\section{Catch the wave}

Noboru Suzuki and colleagues have characterized an unusual spontaneous mouse mutant (Proc. Natl. Acad. Sci. USA 100, 96809685; 2003). The mutant

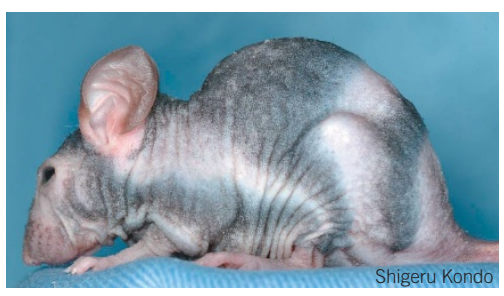
mouse, Foxn $1^{\text {tw }}$, has a defect in the splicing of Foxn1, a gene that is required for hair follicle development. Remarkably, the Foxn $1^{\text {tw }}$ homozygous mice develop sagittal pigmented stripes on their skin that proceed from the armpit in 30-day cycles. Sections of Foxn $1^{\text {tw }}$ skin show that although the mouse is hairless, hair follicles at the wave front are in the activated (anagen) phase and accumulate black pigment, whereas follicles in advance of and behind the wave front are in the resting (telogen) phase. The authors note that this wave pattern resembles that generated by simple chemicals in an excitable medium as a result of the Belousov-Zhabotinsky (BZ) reaction. They conclude that the Foxn $1^{\text {tw }}$ mouse is a biological example of a basic principle in nonbiological pattern formation, because the skin of these mice seems to fulfill all the requirements of the $\mathrm{BZ}$ reaction: it can act as an 'oscillating medium' (the hair follicle cycle guarantees this), it has a 'lateral activator' (sonic hedgehog is a good candidate), and it has a 'pacemaker region' (the armpit).

\section{An essential role for Trf1}

Negative regulators of telomerase include TRF1, TIN2 and PINX1, each of which seems to have a role in telomere length homeostasis. Surprisingly, Jan Karlseder and colleagues have now shown that mice with a targeted deletion of exon 1 of Terf1 (encoding Trf1) have an early embryonic growth defect and die at embryonic day (E) 5.5-6.5 (Mol. Cell. Biol. 23, 6533-6541; 2003). Most embryos carrying a homozygous targeted deletion of Terf1 exon 1 were resorbed by E6.5 and had abnormal ectoplacental cones and extraembryonic structures, as well as apoptotic death of cells in the inner cell mass. The absence of p53, which is part of the cellular response to dysfunctional telomeres, failed to rescue the embryos, although loss of p53 did extend the viability of many embryos until E7.5-8.5. Telomerase deficiency also failed to rescue the embryos, suggesting that the embryonic lethality is largely independent of effects on telomere length. Karlseder et al. suggest that Trf1 might be required for embryonic stem cell proliferation, consistent with the fact that $\operatorname{Tr} f 1$ is on the list of genes associated with the 'stem cell phenotype' (Science 298, 597-600; 2002).

\section{In the running with $A C T N 3$}

Researchers in Australia recently reported a polymorphism in ACTN3, encoding $\alpha$-actinin-3, associated with sprint versus endurance performance among elite athletes (Am. J. Hum. Genet. 73, 627-631; 2003). The polymorphism results in either an arginine $(\mathrm{R})$ or a premature stop (X) at position 577 of $\alpha$-actinin-3. The frequency of the $\alpha$ actinin-3-null genotype (two $\mathrm{X}$ alleles) varies from $>1 \%$ to $25 \%$ in natural populations; in Europeans it is $\sim 18 \%$. Its frequency was significantly lower-only 6\%-among elite Australian sprinters. The sprinters also had a significantly higher frequency of the RR genotype

Research Notes written by David Gresham, Monica Harrington, Alan Packer and Michael Stebbins and a lower frequency of the heterozygous RX genotype. In contrast, elite endurance athletes had a slightly higher frequency of the XX genotype. The exact function of $\alpha$-actinin-3 is unknown, but it is the predominant fast fiber isoform in both human and mouse skeletal muscle. It may promote the formation of fast-twitch muscle fibers or alter glucose metabolism. The report raises the idea that genetic differences, such as that in $A C T N 3$, could be useful predictors of athletic performance at the elite level, although such potential has not been established. ACTN3 is not the first gene to be associated with athletic performance; it joins $A C E$, encoding angiotensin-converting enzyme, which also has two variants, one associated with endurance and the other with sprint performance (Nature 393, 221-222; 1998).

$M H$

\section{Inferring function}

Microarrays are a powerful tool for capturing global gene activity and classifying biological states. But claims that expression arrays have provided functional insights are usually founded on shaky logic. Now Joshua Stuart and colleagues (Science advance online publication, 21 August 2003; doi:10.1126/science.1087447) use evolutionary conservation of coexpression to infer the functional relationship between gene products. The authors identified over 6,000 homologs in yeast, worms, flies and humans. Using expression data from some 3,000 published microarray experiments, they sought to identify pairs of genes that were coexpressed in multiple organisms. This resulted in over 20,000 coexpression relationships that are conserved across evolution. The authors argue that the evolutionary conservation of the coexpression pattern for these genes is indicative of a conserved functional relationship. They back up this claim by providing independent validation for a subset of predicted relationships and functions of genes. Conserved coexpressed genes could be further grouped into modules of interacting genes. This study provides a powerful tool for preliminary functional assignment of unannotated genes. It also provides a glimpse of the core biological machinery that is present in evolutionarily diverged organisms.

$D G$

\section{Pheromones and courtship}

In Drosophila, courtship and mating occur in a series of sequential steps that involve all the senses. To detect female pheromones, a male fly taps the abdomen of the female with his forelegs and licks her genitalia. There are a larger number of taste bristles on the male forelegs, each with two or three gustatory receptor neurons that facilitate pheromone recognition. In a recent study, Steven Bray and Hubert Amrein describe the identification of a putative pheromone receptor for Drosophila (Neuron 39, 1019-1029; 2003). The two report that Gr68a, a G-protein-coupled receptor, is expressed in ten male-specific taste bristles in the foreleg, and that ablation of the neurons in which Gr68a is expressed results in a severe reduction of successful copulation. To further test whether the receptors could indeed recognize female pheromones, the researchers overexpressed the Transformer protein in the secretory cells of male flies, resulting in normal male flies expressing female pheromones. These flies were aggressively courted by other male flies. However, flies in which Gr68a-expressing neurons were ablated had greatly reduced male-feminized male courting behavior. Finally, RNAi knockdown of Gr68a transcripts resulted in reduced courtship behavior, indicating an acute need for the receptor for pheromone recognition. Gr68a thus represents the first pheromone receptor that plays a distinct role in courtship behavior. MS 